\title{
Note on a Allen-Cahn equation with Caputo-Fabrizio derivative
}

\author{
Phuong Duc Nguyen \\ Faculty of Fundamental Science, Industrial University of Ho Chi Minh City, Ho Chi Minh City 700000, Vietnam.
}

\begin{abstract}
In this short note, we investigate the Allen-Cahn equation with the appearance of the Caputo-Fabizzio derivative. We obtain a local solution when the initial value is small enough. This is an equation that has many practical applications. The power term in the nonlinear component of the source function and the Caputo-Fabizzio operator combine to make finding the solution space more difficult than the classical problem. We discovered a new technique, connecting Hilbert scale and $L^{p}$ spaces, to overcome these difficulties. Evaluation of the smoothness of the solution was also performed. The research ideas in this paper can be used for many other models.
\end{abstract}

Keywords: Allen-Cahn equation, Fractional diffusion equation; Caputo-Fabizzio, regularity. 2010 MSC: 35R11, 35B65, 26A33

\section{Introduction}

Let $\mathcal{D}$ be a $\mathcal{C}^{2}$ bounded open set of $\mathbb{R}^{N}$ with sufficient smooth boundary and $T>0$. In this paper, we consider the fractional Sobolev equation

$$
\left\{\begin{array}{l}
\mathrm{CF}_{t}^{\alpha} u=\Delta u+u-u^{3}, \quad(x, t) \in \mathcal{D} \times(0, T), \\
u=0, \quad(x, t) \in \partial \mathcal{D} \times(0, T), \\
u(x, 0)=u_{0}(x), \quad x \in \mathcal{D}
\end{array}\right.
$$

where $\mathrm{CF}_{t} D_{t}^{\alpha}$ is the Caputo-Fabrizio operator for fractional derivatives of order $\alpha$ which is defined as (see [24])

$$
\mathrm{CF}_{t}^{\alpha} v(t)=\frac{H(\alpha)}{1-\alpha} \int_{0}^{t} \mathcal{D}_{\alpha}(t-s) \frac{\partial v(s)}{\partial s} \mathrm{~d} s, \quad \text { for } t \geq 0
$$

Email address: nguyenducphuong@iuh.edu.vn (Phuong Duc Nguyen) 
where we denote by the kernel $\mathcal{D}_{\alpha}(z)=\exp \left(-\frac{\alpha}{1-\alpha} z\right)$ and $H(\alpha)$ satisfies $H(0)=H(1)=1$, (see e.g. [22, 23]). The main goal of this note is to prove the existence of a local solution to the problem given the input data $u_{0}$ in the space $L^{p}$ space. If $\alpha=1$, Problem 1.1) is called Allen-Cahn equation with classical derivative. If we replace Caputo-Fabizzio derivative by Caputo derivative, Problem (1.1) is studied by [28]. Allen-Cahn model was originally introduced to transform the description of boundaries in coherent solids [29].

Fractional calculus has a long history and has many applications in simulations of physical phenomena or real life for example, mechanics, electricity, chemistry, biology,.. Many mathematical models cannot be expressed in terms of classical derivatives because of the effects of external forces. Therefore, the introduction of fractional calculus has important implications for modeling physical and engineering processes in cases where classical derivatives are not available. Some works are attracting the attention of the community for fractional differential equations, like A. Debbouche and his group [4, 5, 6], E. Karapınar et al [11, 12, 13, 14, 15, 16, 17, 18]. The Caputo-Fabrizio fractional derivative was first introduce by [22] which makes sense to avoid singular kernels. It is detemined by the convolution of the exponential function and the first order derivative. This operator has been widely applied to a number of derivative modes in many fields, such as biology, physics, control systems [19, 20, 26].

There are two main challenges and difficulties when we consider this problem. The first difficulty is that it is difficult for us to apply the $L^{p}$ estimate to the semigroup heat operators because of the appearance of the Caputo-Fabizzio operator. Indeed, in F. Weisler's work [27], they have the advantage of using the $L^{p}$ evaluation for the half heat group where we do not apply. The second challenge is that we cannot evaluate the function $u^{p}$ on Hilbert scale spaces but can only estimates on $L^{p}$ while the Caputo-Fabizzio operator can only handle in Hilbert scale space. Those are the hard points that we need to overcome. Our novel idea is to connect the evaluations together by embeddings between $L^{p}$ and Hilbert scales. This new technique can be applied to prove the existence of solutions to a wide range of problems.

\section{Main results}

Before giving the main result, we recall some knowledge about function spaces and embeddings. Note that $A=-\Delta$ is a symmetric uniformly elliptic operator, hence it possesses a non-negative, non-decreasing and discrete spectrum $0<\lambda_{1} \leq \lambda_{2} \leq \ldots \leq \lambda_{n} \nearrow \infty$. The corresponding eigenvectors of $A$ are denoted by $e_{n} \in D(A)$, which satisfy $A e_{n}(x)=\lambda_{n} e_{n}(x)$ for $x \in \mathcal{D}$. Let us introduce the Hilbert scale space, which is given as follows

$$
\mathcal{H}^{r}(\mathcal{D})=\left\{f \in L^{2}(\mathcal{D}), \quad \sum_{n=1}^{\infty} \lambda_{n}^{2 r}\left\langle f, e_{n}\right\rangle_{L^{2}(\mathcal{D})}^{2}<\infty\right\},
$$

for any $r \geq 0$. Here the symbol $\langle\cdot, \cdot\rangle_{L^{2}(\mathcal{M})}$ denotes the inner product in $L^{2}(\mathcal{M})$. It is well-known that $\mathcal{H}^{r}(\mathcal{D})$ is a Hilbert space corresponding to the following norm

$$
\|f\|_{\mathcal{H}^{r}(\mathcal{D})}=\sqrt{\sum_{n=1}^{\infty} \lambda_{n}^{2 r}\left\langle f, e_{n}\right\rangle_{L^{2}(\mathcal{D})}^{2}}, \quad f \in \mathcal{H}^{r}(\mathcal{D}) .
$$

In view of $\mathcal{H}^{r}(\mathcal{D}) \equiv D\left((-\mathbb{A})^{r}\right)$ is a Hilbert space. Then $D\left((-\mathbb{A})^{-r}\right)$ is a Hilbert space with the norm

$$
\|v\|_{D\left((-\mathbb{A})^{-r}\right)}=\left(\sum_{n=1}^{\infty}\left|\left\langle v, e_{n}\right\rangle\right|^{2} \lambda_{j}^{-2 r}\right)^{\frac{1}{2}},
$$

where $\langle\cdot, \cdot\rangle$ in the latter equality denotes the duality between $D\left((-\mathbb{A})^{-r}\right)$ and $D\left((-\mathbb{A})^{r}\right)$. 
Lemma 1. The following inclusions hold true:

$$
\begin{aligned}
& L^{p}(\Omega) \hookrightarrow D\left(\mathcal{A}^{\sigma}\right), \quad \text { if } \quad-\frac{N}{4}<\sigma \leq 0, \quad p \geq \frac{2 N}{N-4 \sigma}, \\
& \left.D\left(\mathcal{A}^{\sigma}\right) \hookrightarrow L^{p}(\Omega), \quad \text { if } \quad 0 \leq \sigma<\frac{N}{4}, \quad p \leq \frac{2 N}{N-4 \sigma} .\right\}
\end{aligned}
$$

Definition 1. The function $v$ is called a mild solution of Problem 1.1 if it satisfies that

$$
v(t)=\mathbf{S}_{\alpha}(t) u_{0}+\int_{0}^{t} \mathbf{S}_{\alpha}(t-s) G(v(s)) d s
$$

where $G(v)=v-v^{3}$ and $\mathbf{S}_{\alpha}(t)$ is defined by

$$
\mathbf{S}_{\alpha}(t) f=\left(1+\bar{\alpha} \lambda_{n}\right)^{-1} \exp \left(\frac{-\alpha \lambda_{n}}{1+\bar{\alpha} \lambda_{n}} t\right)\left\langle f, e_{n}\right\rangle_{L^{2}(\mathcal{D})} e_{n}(x), \quad \bar{\alpha}=1-\alpha .
$$

for any $w \in L^{2}(\mathcal{D})$.

Lemma 2. Let $f \in H^{m-2}(\mathcal{D})$. Then

$$
\left\|\mathbf{S}_{\alpha}(t) f\right\|_{H^{m}(\mathcal{D})} \leq \frac{1}{1-\alpha}\|f\|_{H^{m-2}(\mathcal{D})} .
$$

Proof. Using Parseval's equality, we get that

$$
\begin{aligned}
\left\|\mathbf{S}_{\alpha}(t) f\right\|_{H^{m}(\mathcal{D})} & =\left(\sum_{n=1}^{\infty} \lambda_{n}^{m}\left(1+\bar{\alpha} \lambda_{n}\right)^{-2} \exp \left(\frac{-2 \alpha \lambda_{n}}{1+\bar{\alpha} \lambda_{n}} t\right)\left\langle f, e_{n}\right\rangle_{L^{2}(\mathcal{D})}^{2}\right)^{1 / 2} \\
& \leq \frac{1}{\bar{\alpha}}\left(\sum_{n=1}^{\infty} \lambda_{n}^{m-2}\left\langle f, e_{n}\right\rangle_{L^{2}(\mathcal{D})}^{2}\right)^{1 / 2}=\frac{1}{1-\alpha}\|f\|_{H^{m-2}(\mathcal{D})}
\end{aligned}
$$

Remark 1. The hardest part about proving the theorem is that we don't immediately get the $L^{p}$ estimate for the operator $\mathbf{S}_{\alpha}(t)$. For classical problem, we are available for apply $L^{p}$ estimate since the ideas of [27]. However, we face the operator $\mathbf{S}_{\alpha}(t)$ as above, we have difficulty things for considering $L^{p}$ estimate. The second difficulty is that we cannot evaluate the source function on Hilbert scales space.

Theorem 1. Let $u_{0} \in L^{q / 3}(\mathcal{D})$ where $2 \leq q \leq 6$ and $q \geq 2 N$. Then problem 1.1) has a local mild solution $u \in \mathbf{X}_{\beta, q} \cap L^{p}\left(0, T ; L^{q}(\mathcal{D})\right)$ where $0<\beta<1 / 3$ and $1<p<\frac{1}{\beta}$.

Remark 2. Since the assumption $2 \leq q \leq 6$ and $q \geq 2 N$, we can see that $1 \leq N \leq 3$. Hence, we only study the local existence for the dimensional of the domain $\mathcal{D}$ is about 1 to 3 .

Proof. It is obvious to see that

$$
|G(u)-G(v)|=\left|(u-v)-\left(u^{3}-v^{3}\right)\right| \leq 2(|u-v|)\left(1+|u|^{2}+|v|^{2}\right) .
$$

Using Hölder inequality, we continue to get the following estimate

$$
\begin{aligned}
& \left\|G\left(v_{1}\right)-G\left(v_{2}\right)\right\|_{L^{\frac{q}{3}(\mathcal{D})}}=\left(\int_{D}\left|G\left(v_{1}\right)-G\left(v_{2}\right)\right|^{\frac{q}{3}} d x\right)^{\frac{3}{q}} \\
& \leq 2\left(\int_{\mathcal{D}}\left|\left(\left|v_{1}-v_{2}\right|\right)\left(\left|v_{1}-v_{2}\right|^{2}+\left|v_{1}-v_{2}\right|^{2}\right)\right|^{\frac{q}{3}} d x\right)^{\frac{3}{q}}
\end{aligned}
$$




$$
\begin{aligned}
& \leq 2\left(\int_{\mathcal{D}}\left(\left|v_{1}-v_{2}\right|^{\frac{q}{3}}\right)^{3}\right)^{\frac{1}{3} \frac{3}{q}}\left(\int_{\mathcal{D}}\left(\left(\left|v_{1}\right|^{2}+\left|v_{2}\right|^{2}\right)^{\frac{q}{3}}\right)^{\frac{3}{2}}\right)^{\frac{2}{3} \frac{3}{q}} \\
& \leq 2\left\|v_{1}-v_{2}\right\|_{L^{\rho}(\mathcal{D})}\left[\left(\int_{\mathcal{D}}\left(\left|v_{1}\right|^{q}\right)^{\frac{2}{q}}+\int_{D}\left(\left|v_{2}\right|^{q}\right)^{\frac{2}{q}}\right]\right. \\
& =2\left\|v_{1}-v_{2}\right\|_{L^{q}(\mathcal{D})}\left[\left\|v_{1}\right\|_{L^{q}(\mathcal{D})}^{2}+\left\|v_{2}\right\|_{L^{q}(\mathcal{D})}^{2}\right] .
\end{aligned}
$$

Define the Banach space $\mathbf{X}_{\beta, q}$ of all Bochner integrable functions $u:[0, T] \rightarrow L^{q}(\mathcal{D})$ such that $t^{\varepsilon} u$ are bounded continuous functions, endowed with the norm

$$
\sup _{0 \leq t \leq T} t^{\beta}\|u(t, .)\|_{L^{q}(\mathcal{D})}<\infty .
$$

Let the function $J$ be as follows

$$
J v(t)=\mathbf{S}_{\alpha}(t) u_{0}+\int_{0}^{t} \mathbf{S}_{\alpha}(t-s) G(v(s)) d s
$$

Let $v, w \in \mathbf{X}_{\beta, q}$. Since Sobolev embedding $\mathcal{H}^{\frac{N q-2 N}{4 q}}(\mathcal{D}) \hookrightarrow L^{q}(\mathcal{D})$ for any $q>2$, we get that

$$
\begin{aligned}
\|J v-J w\|_{\mathbf{X}_{\beta, q}} & =\sup _{0 \leq t \leq T} t^{\beta}\|J v(., t)-J w(., t)\|_{L^{q}(\mathcal{D})} \\
& \leq \sup _{0 \leq t \leq T} t^{\beta}\|J v(., t)-J w(., t)\|_{\mathcal{H}^{\frac{N q-2 N}{4 q}}(\mathcal{D})}
\end{aligned}
$$

It is obvious to see that

$$
\begin{aligned}
& \left\|\int_{0}^{t} \mathbf{S}_{\alpha}(t-s) G(v(s)) d s-\int_{0}^{t} \mathbf{S}_{\alpha}(t-s) G(w(s)) d s\right\|_{\mathcal{H}^{\frac{N q-2 N}{4 q}}(\mathcal{D})} \\
& \leq \frac{1}{1-\alpha} \int_{0}^{t}\|G(v(s))-G(w(s))\|_{\mathcal{H}^{\frac{N q-2 N}{4 q}-2}(\mathcal{D})} d s \\
& \leq C(N, q) \int_{0}^{t}\|G(v(s))-G(w(s))\|_{\mathcal{H}^{\frac{N q-6 N}{4 q}}(\mathcal{D})} d s
\end{aligned}
$$

where we note that

$$
\mathcal{H}^{\frac{N q-6 N}{4 q}}(\mathcal{D}) \hookrightarrow \mathcal{H}^{\frac{N q-2 N}{4 q}-2}(\mathcal{D})
$$

since the condition $q \geq 2 N$.

Based on Sobolev embedding $L^{\frac{q}{3}}(\mathcal{D}) \hookrightarrow \mathcal{H}^{\frac{N q-6 N}{4 q}}(\mathcal{D})$ for any $q<6$, we derive that

$$
\int_{0}^{t}\|G(v(s))-G(w(s))\|_{\mathcal{H}^{\frac{N q-6 N}{4 q}}(\mathcal{D})} d s \leq C(N, q) \int_{0}^{t}\|G(v(s))-G(w(s))\|_{L^{\frac{q}{3}(\mathcal{D})}} d s
$$

Set the following ball

$$
B(R):=\left\{w:[0, T] \rightarrow L^{q}(\mathcal{D}),\|w\|_{\mathbf{X}_{\beta, q}} \leq R\right\}
$$

If $v, w \in \mathbf{X}_{\beta, q}$ then we get

$$
\|v(., s)\|_{L^{q}(\mathcal{D})} \leq s^{-\beta}\|v\|_{\mathbf{X}_{\beta, q}} \leq s^{-\beta} R .
$$

In view of 2.6, we obtain

$$
\int_{0}^{t}\|G(v(s))-G(w(s))\|_{L^{\frac{q}{3}(\mathcal{D})}} d s
$$




$$
\begin{aligned}
& \leq 2 \int_{0}^{t}\|v(., s)-w(., s)\|_{L^{q}(\mathcal{D})}\left[\|v(., s)\|_{L^{q}(\mathcal{D})}^{2}+\|w(., s)\|_{L^{q}(\mathcal{D})}^{2}\right] \\
& \leq 4 R^{2} \int_{0}^{t} s^{-2 \beta}\|v(., s)-w(., s)\|_{L^{q}(\mathcal{D})} d s \\
& =4 R^{2} \int_{0}^{t} s^{-3 \beta} s^{\beta}\|v(., s)-w(., s)\|_{L^{q}(\mathcal{D})} d s \leq 4 R^{2}\left(\int_{0}^{t} s^{-3 \beta} d s\right)\|v-w\|_{\mathbf{X}_{\beta, q}}
\end{aligned}
$$

Under the condition $\beta<1 / 3$, we know that $\int_{0}^{t} s^{-3 \beta} d s$ is convergent. So, we get that

$$
\int_{0}^{t}\|G(v(s))-G(w(s))\|_{L^{\frac{q}{3}(\mathcal{D})}} d s \leq 4 R^{2} \frac{t^{1-3 \beta}}{1-3 \beta}\|v-w\|_{\mathbf{X}_{\beta, q}} .
$$

Combining (2.8), 2.9), 2.14), we arrive at

$$
\begin{aligned}
\|J v-J w\|_{\mathbf{X}_{\beta, q}} & \leq\left(\sup _{0 \leq t \leq T} t^{\beta+1-3 \beta}\right) C(N, q) 4 R^{2} \frac{1}{1-3 \beta}\|v-w\|_{\mathbf{X}_{\beta, q}} \\
& \leq \frac{4 C(N, q) R^{2} T^{1-2 \beta}}{1-3 \beta}\|v-w\|_{\mathbf{X}_{\beta, q}} .
\end{aligned}
$$

Let us choose $R, T$ such that

$$
\frac{4 C(N, q) R^{2} T^{1-2 \beta}}{1-3 \beta}<1 / 2
$$

We next evaluate

$$
\left\|\mathbf{S}_{\alpha} u_{0}\right\|_{\mathbf{X}_{\beta, q}}=\sup _{0 \leq t \leq T} t^{\beta}\left\|\mathbf{S}_{\alpha}(t) u_{0}\right\|_{L^{q}(\mathcal{D})} \leq C(N, q) \sup _{0 \leq t \leq T} t^{\beta}\left\|\mathbf{S}_{\alpha}(t) u_{0}\right\|_{\mathcal{H}^{\frac{N q-2 N}{4 q}}(\mathcal{D})} .
$$

By looking back Lemma (2), we find that

$$
\left\|\mathbf{S}_{\alpha}(t) u_{0}\right\|_{\mathcal{H}}^{\frac{N q-2 N}{4 q}(\mathcal{D})} \leq \frac{1}{1-\alpha}\left\|u_{0}\right\|_{\mathcal{H}} \frac{N q-2 N-8 q}{4 q}(\mathcal{D})
$$

Since the condition $q \geq 2 N$, we remind the Sobolev embedding

$$
\mathcal{H}^{\frac{N q-6 N}{4 q}}(\mathcal{D}) \hookrightarrow \mathcal{H}^{\frac{N q-2 N-8 q}{4 q}}(\mathcal{D})
$$

. This allows us to provide that the following estimate

$$
\left\|\mathbf{S}_{\alpha} u_{0}\right\|_{\mathbf{X}_{\beta, q}} \leq \frac{C(N . q)}{1-\alpha}\left\|u_{0}\right\|_{\mathcal{H}^{\frac{N q-6 N}{4 q}}(\mathcal{D})}
$$

The condition $q<6$ give us the embedding

$$
L^{\frac{q}{3}}(\mathcal{D}) \hookrightarrow \mathcal{H}^{\frac{N q-6 N}{4 q}}(\mathcal{D})
$$

which leads to

$$
\left\|u_{0}\right\|_{\mathcal{H}^{\frac{N q-6 N}{4 q}}(\mathcal{D})} \leq C(N, q)\left\|u_{0}\right\|_{L^{\frac{q}{3}(\mathcal{D})}}
$$

Hence, we deduce that

$$
\|J(\mathbf{0})\|_{\mathbf{X}_{\beta, q}}=\left\|\mathbf{S}_{\alpha} u_{0}\right\|_{\mathbf{X}_{\beta, q}} \leq T^{\beta} C(N, q)\left\|u_{0}\right\|_{L^{\frac{q}{3}(\mathcal{D})}} .
$$


Let us choose $T$ such that

$$
T^{\beta} C(N, q)\left\|u_{0}\right\|_{L^{\frac{q}{3}(\mathcal{D})}} \leq \frac{R}{2}
$$

It follows from 2.15 that

$$
\begin{aligned}
\|J v\|_{\mathbf{X}_{\beta, q}} & \leq\|J v-J(\mathbf{0})\|_{\mathbf{X}_{\beta, q}}+\|J(\mathbf{0})\|_{\mathbf{X}_{\beta, q}} \\
& \leq \frac{1}{2}\|v\|_{\mathbf{X}_{\beta, q}}+T^{\beta} C(N, q)\left\|u_{0}\right\|_{L^{\frac{q}{3}}(\mathcal{D})}
\end{aligned}
$$

for any $v \in \mathbf{X}_{\beta, q}$. This says that

$$
\|J v\|_{\mathbf{X}_{\beta, q}}<R
$$

which allows us to deduce that $J$ is a mapping from $B(R)$ to itself $B(R)$. Using the Banach mapping theorem, we conclude that $J$ have a fixed point $u$ in $B(R)$.

Our aim is to investigate the regularity of the mild solution $u$. Indeed, we get

$$
\|u\|_{\mathbf{X}_{\beta, q}} \leq\|J u-J(\mathbf{0})\|_{\mathbf{X}_{\beta, q}}+\|J(\mathbf{0})\|_{\mathbf{X}_{\beta, q}} \leq \frac{1}{2}\|u\|_{\mathbf{X}_{\beta, q}}+T^{\beta} C(N, q)\left\|u_{0}\right\|_{L^{\frac{q}{3}}(\mathcal{D})} .
$$

This implies that

$$
\|u\|_{\mathbf{X}_{\beta, q}} \leq 2 T^{\beta} C(N, q)\left\|u_{0}\right\|_{L^{\frac{q}{3}(\mathcal{D})}} \cdot
$$

Hence, we find that

$$
\|u(., t)\|_{L^{q}(\mathcal{D})} \leq 2 T^{\beta} t^{-\beta} C(N, q)\left\|u_{0}\right\|_{L^{\frac{q}{3}(\mathcal{D})}}
$$

The above expression allows us to obtain that

$$
\left(\int_{0}^{T}\|u(., t)\|_{L^{q}(\mathcal{D})}^{p} d t\right)^{1 / p} \leq 2 T^{\beta} C(N, q)\left\|u_{0}\right\|_{L^{\frac{q}{3}(\mathcal{D})}}\left(\int_{0}^{T} t^{-\beta p} d t\right)^{1 / p}
$$

Since $1<p<\frac{1}{\beta}$, we deduce that the proper integral $\int_{0}^{T} t^{-\beta p} d t$ is convergent. Therefore, we can say that $u \in L^{p}\left(0, T ; L^{q}(\mathcal{D})\right)$.

\section{References}

[1] N.H. Tuan, Y. Zhou, T.N. Thach, N.H. Can, Initial inverse problem for the nonlinear fractional Rayleigh-Stokes equation with random discrete data Commun. Nonlinear Sci. Numer. Simul. 78 (2019), 104873, 18 pp.

[2] N.H. Tuan, L.N. Huynh, T.B. Ngoc, Y. Zhou, On a backward problem for nonlinear fractional diffusion equations Appl. Math. Lett. 92 (2019), 76-84.

[3] T.B. Ngoc, Y. Zhou, D. O'Regan, N.H. Tuan, On a terminal value problem for pseudoparabolic equations involving Riemann-Liouville fractional derivatives, Appl. Math. Lett. 106 (2020), 106373, 9 pp.

[4] J. Manimaran, L. Shangerganesh, A. Debbouche, Finite element error analysis of a time-fractional nonlocal diffusion equation with the Dirichlet energy J. Comput. Appl. Math. 382 (2021), 113066, 11 pp

[5] J. Manimaran, L. Shangerganesh, A. Debbouche, A time-fractional competition ecological model with cross-diffusion Math. Methods Appl. Sci. 43 (2020), no. 8, 5197-5211

[6] N.H. Tuan, A. Debbouche, T.B. Ngoc, Existence and regularity of final value problems for time fractional wave equations Comput. Math. Appl. 78 (2019), no. 5, 1396-1414.

[7] I. Podlubny, Fractional differential equations, Academic Press, London, 1999.

[8] B. D. Coleman, W. Noll, Foundations of linear viscoelasticity, Rev. Mod. Phys., 33(2) 239 (1961).

[9] P. Clément, J.A. Nohel, Asymptotic behavior of solutions of nonlinear volterra equations with completely positive kernels, SIAM J. Math. Anal., 12(4) (1981), pp. 514-535. 
[10] X.L. Ding, J.J. Nieto, Analytical solutions for multi-term time-space fractional partial differential equations with nonlocal damping terms, Frac. Calc. Appl. Anal. 21 (2018), pp. 312-335.

[11] R. S. Adiguzel, U. Aksoy, E. Karapınar, I.M. Erhan, On the solution of a boundary value problem associated with a fractional differential equation Mathematical Methods in the Applied Sciences https://doi.org/10.1002/mma.665

[12] H. Afshari, ., Karapınar, A discussion on the existence of positive solutions of the boundary value problems via-Hilfer fractional derivative on b-metric spaces, Advances in Difference Equations volume 2020, Article number: 616 (2020)

[13] H.Afshari, S. Kalantari, E. Karapınar; Solution of fractional differential equations via coupled fixed point, Electronic Journal of Differential Equations, Vol. 2015 (2015), No. 286, pp. 1-12

[14] B. Alqahtani, H. Aydi, E. Karapınar, V. Rakocevic, A Solution for Volterra Fractional Integral Equations by Hybrid Contractions Mathematics 2019, 7, 694.

[15] E. Karapinar, A. Fulga, M. Rashid, L. Shahid, H. Aydi, Large Contractions on Quasi-Metric Spaces with an Application to Nonlinear Fractional Differential-Equations Mathematics 2019, 7, 444.

[16] A.Salim, B. Benchohra, E. Karapınar, J.E. Lazreg, Existence and Ulam stability for impulsive generalized Hilfer-type fractional differential equations Adv Differ Equ 2020, 601 (2020)

[17] E. Karapınar, T.Abdeljawad, F. Jarad, Applying new fixed point theorems on fractional and ordinary differential equations, Advances in Difference Equations, 2019, 2019:421

[18] A. Abdeljawad, R.P. Agarwal, E. Karapınar, P.S. Kumari, Solutions of he Nonlinear Integral Equation and Fractional Differential Equation Using the Technique of a Fixed Point with a Numerical Experiment in Extended b-Metric Space Symmetry 2019, 11, 686 .

[19] N. Hung, H. Binh, N. Luc, A. Nguyen Thi Kieu, L. Long, Stochastic sub-diffusion equation with conformable derivative driven by standard Brownian motion. Advances in the Theory of Nonlinear Analysis and its Application, (2021) 5(3), 287-299

[20] V. Tri, Existence of an initial value problem for time-fractional Oldroyd-B fluid equation using Banach fixed point theorem. Advances in the Theory of Nonlinear Analysis and its Application, (2021) 5 (4), 523-530

[21] N.H. Tuan, N.H. Can, R. Wang, Y. Zhou, Initial value problem for fractional Volterra integro-differential equations with Caputo derivative, Discrete and Continuous Dynamical Systems - B, doi: 10.3934/dcdsb.2021030

[22] M. Caputo, M. Fabrizio, A new definition of fractional derivative without singular kernel, Progr. Fract. Differ. Appl., 1(2) (2015), pp. 1-13.

[23] M. Caputo, M. Fabrizio, Applications of new time and spatial fractional derivatives with exponential kernels, Progr. Fract. Differ. Appl., 2(2) (2016), pp. 1-11.

[24] J. Losada, J.J. Nieto, Properties of a new fractional derivative without singular kernel, Prog. Fract. Differ. Appl., 1(2) (2015), pp. 87-92.

[25] M.S. Hashemi, E. Darvishi, M. Inc, A geometric numerical integration method for solving the Volterra integro-differential equations Int. J. Comput. Math. 95 (2018), no. 8, 1654-1665.

[26] T.M. Atanacković, S. Pillipović, D. Zorica, Properties of the Caputo-Fabrizio fractional derivative and its distributional settings, Fract. Calc. Appl. Anal., 21, (2018), pp. 29-44.

[27] F.B. Weissler, Semilinear evolution equations in Banach spaces J. Functional Analysis 32 (1979), no. 3, 277-296

[28] Q. Du, J. Yang, Z. Zhou, Time-fractional Allen-Cahn equations: analysis and numerical methods J. Sci. Comput. 85 (2020), no. 2, Paper No. 42, 30 pp

[29] S.M. Allen, J.W. Cahn, A microscopic theory for antiphase boundary motion and its application to antiphase domain coarsening, Acta Metall. 27 (6) (1979) 1085-1095 